\title{
COMPARISON STUDY OF CLINICAL MEASUREMENTS AND MONTE CARLO METHOD ON BACKSCATTERED RADIATION DOSE RATE CHANGES BY DISTANCE IN COMPUTERIZED TOMOGRAPHY (CT) FACILITY DURING HEAD SCANS
}

\author{
Huseyin Ozan Tekin ${ }^{*}$, Umit Kara ${ }^{2}$, Ozlem Ozturk3, \\ Tugba Manici3, E.Ebru Altunsoy3, Baris Cavli4
}

\begin{abstract}
${ }^{1}$ Üsküdar University, Vocational School of Health Services, Radiotherapy Department, İstanbul, Turkey ${ }^{2}$ Süleyman Demirel University, Vocational School of Health Services, Medical Imaging Department, Isparta, Turkey 3Üsküdar University, Medical Radiation Research Center (USMERA), İstanbul, Turkey 4Affidea, İstanbul, Turkey
\end{abstract}

\begin{abstract}
A Computerized Tomography (CT) scan combines a series of X-ray images obtained from different angles to create the patient's cross-sectional images of body parts. It can be used especially for bones, blood vessels and soft tissues. As a comparison between two devices, the obtained information by using CT scan on the patient is more significant than the information obtained by a normal plain $X$-ray device. Since CT uses various doses of $X$-rays for the imaging of the body, radiation protection became a major topic of investigation. One of the fundamental principles of radiation protection is the distance factor. In CT facilities, the maximum dose occurs near the gantry and the dose rate decreases by distance. In order to know the rate of the reduction of the amount of dose, that distance is very important for the radiation protection procedure, especially for applying the criteria of the International Commission on Radiological Protection (ICRP) on radiation protection. The aim of this study was the validation of the Monte Carlo method for such backscattering studies. In this study, we measured the backscattered dose rates with small distances from the gantry to the exit door and compared them with the Monte Carlo (MC) results. In order to calculate the Monte Carlo results, we also modeled a simulation input for the CT facility by using MCNP-X (version 2.4.O) Monte Carlo code. We obtained the backscattered dose rate changes that depend on the distance factor by using the Monte Carlo method. We achieved a good agreement between MCNP-X results and clinical experimental results. It can be concluded that Monte Carlo (MC) is a strong tool for radiation protection calculations in CT facilities.
\end{abstract}

Key words: Monte Carlo, $M C N P-X, C T$

DOI: $10.21175 /$ RadProc.2016.32

\section{INTRODUCTION}

Computed tomography is an imaging method that produces cross-sectional images, representing in each pixel the local X-ray attenuation properties of the body. Since the introduction of helical computed tomography (CT) in the early 1990's, the technology and capabilities of CT scanners have changed tremendously. By the development of technology on diagnostic devices, procedures are performed with detailed information about the patient. The patient is placed between the source and the detector, and the detector is configured with its geometric center located at the x-ray source. In addition to various benefits of medical imaging by using CT, of course, there are some risk groups associated with the $\mathrm{X}$-ray radiation exposure. The possibility for the absorbed X-rays to cause cancer or inheritable mutations leading to genetically associated diseases in offspring is thought to be very small for radiation doses of the magnitude associated with the CT process. Under some rare circumstances of prolonged, high-dose exposure, $\mathrm{x}$ rays can cause other adverse health effects, such as skin erythema), skin tissue injury, and birth defects following in-utero exposure. Of course, there are some considerable absorbed dose rates from the CT scan. Table 1 shows the dose a patient could receive during the procedure [1]. As we see from Table 1, the values should be considered in terms of which would cause biological effects.

Table 1. Effective dose rates in the examination

\begin{tabular}{|l|l|}
\hline Examination & Effective Dose (mSv) \\
\hline IVP & 2.5 \\
\hline Barium Swallow & 1.5 \\
\hline Barium Enema & 7.0 \\
\hline CT Head & 2.0 \\
\hline CT Chest & 8.0 \\
\hline CT Abdomen & 10.0 \\
\hline CT Pelvis & 10.0 \\
\hline Angioplasty & $7.5-57.0$ \\
\hline Coronary Angiogram & $4.6-15.8$ \\
\hline
\end{tabular}

\footnotetext{
*huseyinozan.tekin@uskudar.edu.tr
} 
High-radiation exposure occurs during computed tomographic (CT) fluoroscopy [2]. This high rate of radiation is a condition that is understandable to the patient because of the imaging procedure, but we have to consider one other case that the radiation dose around a CT device that can affect the CT operator or other staff. For the CT operator, exposure is primarily a function of scattered radiation and collimator or gantry leakage [3]. In the past several years, some work has been done on this subject [4-6]. In recent years, such dosimetry processes have become possible to be performed by using the Monte Carlo (MC) method. Monte Carlo calculations, some investigations and dose calculations by using the patient and phantom have been done with the MC method [7].

\section{MATERIALS AND METHODS}

In this study, we considered our investigations as a two-step process of clinical measurements around CT device and Monte Carlo studies by using MCNP-X general-purpose code. Experimental measurements were obtained in the processes performed by GMOptima 66oCT (128 Slice) device for the same imaging parameters as of CT Head examinations which are often performed by $120 \mathrm{kV}$. We obtained the measurements for 9 different locations - from the CT gantry to the exit door. Figure 1 represents the measurement locations according to the position of the gantry.

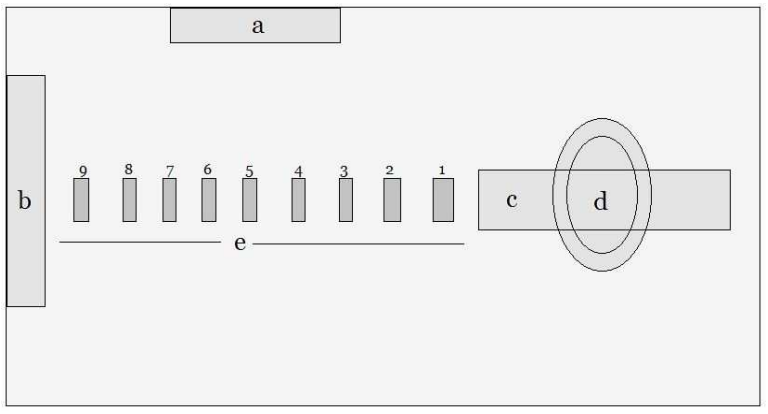

Figure 1. Schematic view of CT room

In Figure 1, $a$ is the location of the patient door, $b$ is the location of the control room for the operators, $c$ is the location of the patient table and $d$ is the gantry of the CT device. From Figure 1, we also can see the measurement locations which are marked with numbers from 1 to 9 . The exact values of measurement locations are given in Table 2 in the ID. column with measured dose rates, respectively. All the dose measurements are performed by using the Polimaster Survey Meter (PM1405), shown in Figure 2, a compact multifunction instrument capable for measuring X-ray, gamma and beta radiation. We performed the simultaneous and selective measurement of the x-ray radiation intensity by using $\mathrm{PM} 1405$ during Head CT examinations. We would like to state that all the measurements were performed by using the user interface of PM1405 in the operating room by remote control with a personal computer which comes with detector as an install driver by considering radiation protection justification.

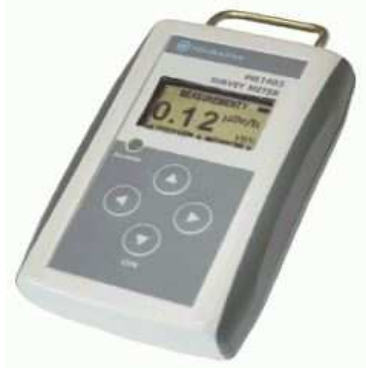

Figure 2. Polimaster Survey Meter PM1405

As a next step in the study, we calculated the x-ray intensities by using the MCNP-X code. MCNP-X (Monte Carlo N-Particle eXtended) is a general purpose Monte Carlo radiation transport code for modeling the interaction of radiation with materials. MCNP-X is fully three-dimensional and it utilizes extended nuclear cross section libraries and uses physics models for particle types. MCNP-X is used in nuclear medicine, radiation physics, nuclear safeguards, accelerator applications, nuclear criticality, etc. It also allows the various specific working areas in simulations. One of them is modeling of the detector geometry. The detector geometry is modeled with the MCNP-X code, which allows the simulation of physical events occurring during the detection and it registers them to build energy spectra. MCNP-X is also used or calculating the flux at a point or ring named $\mathrm{F}_{5}$ tally. For each source particle and each collision event, a deterministic estimate is made of the fluence contribution at the detector point.

\section{RESULTS}

We performed measurements in nine different distances, respectively, by increasing the distance by 50 $\mathrm{cm}$ in each measurement. We considered the average dose rates in each distance. Table 2 gives the examination and dose values for different distances and dose rates.

Table 2. Distance and dose rates

\begin{tabular}{|l|c|c|c|c|}
\hline Examination & $\begin{array}{c}\text { CT } \\
\text { Dose } \\
(\mathrm{kV})\end{array}$ & Distance & $\begin{array}{c}\text { Measured } \\
\text { Dose } \\
(\mathrm{msV} / \mathrm{h})\end{array}$ & ID. \\
\hline CT Head & 120 & 20 & 26,77 & 1 \\
\hline CT Head & 120 & 70 & 3,59 & 2 \\
\hline CT Head & 120 & 120 & 5,38 & 3 \\
\hline CT Head & 120 & 170 & 3,12 & 4 \\
\hline CT Head & 120 & 220 & 1,41 & 5 \\
\hline CT Head & 120 & 270 & 0,64 & 6 \\
\hline CT Head & 120 & 320 & 0,57 & 7 \\
\hline CT Head & 120 & 370 & 0,44 & 8 \\
\hline CT Head & 120 & 420 & 0,26 & 9 \\
\hline
\end{tabular}

Gamma radiation is a part of the electromagnetic spectrum. A gamma ray is not absorbed by the air, but its intensity decreases because it spreads out. 
Accordingly, the intensity changes with the inverse square of the distance: it follows an inverse square law. As we see from Table 1, the dose rates decrease by the increase of the distance from CT gantry. By considering the clinical parameters, we also calculated the changes of the dose rate by using MCNP-X. In our calculations, we used $10^{6}$ particles as the number of particles. In our calculations, we also used the Intel Core $930-2.80$ $\mathrm{GHz} 64$ bit operating system as a simulation device. As we see from Figure 3, we measured and calculated values compared in the same graph to see statistical differences.

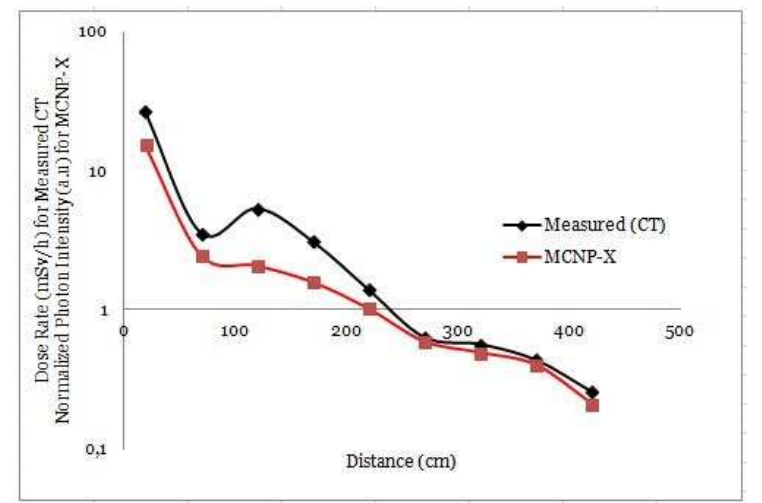

Figure 3. Comparison of measurement and calculation

Since some special experimental conditions such as the presence of material which creates the effect of backscattering can occur during the measurements, we can see from Figure 3 that there is a remarkable increase of the dose rate for around $120 \mathrm{~cm}$. As a discussion subject, we can emphasize a possible backscattering for the related distance. Since Monte Carlo simulates all the interactions by considering the framework of formulization and interaction within the material, the same value difference was not observed in the MCNP-X calculation.

\section{CONCLUSION}

For decades, studies on Monte Carlo applications in medical studies have achieved great development, especially by the development of different Monte Carlo codes. By considering real clinical conditions, we can mention some small result differences. Of course, any small clinical device can cause different backscattering in a room. Aim of this study was to validation of Monte Carlo method for such backscattering studies. It can be concluded that Monte Carlo is a strong tool for backscattered dose rate calculations and by considering the results of this study, it can be also concluded that this method can also be used for future clinical size and radiation protection studies, conducted in discussion with ICRP and IAEA for better shielding in radiology clinics.

\section{REFERENCES}

1. B.F. Wall, D. Hart, "Revised radiation doses for typical $\mathrm{X}$-ray examinations. Report on a recent review of doses to patients from medical X-ray examinations in the UK by NRPB. National Radiological Protection Board," Brit. J. Radiol., vol. 70, pp. 437-439, 1997

2. Z. Neeman, S.A. Dromi, S. Sarin and B.J. Wood, "CT Fluoroscopy Shielding Decreases in Scattered Radiation for the Patiendt and Operator," J. Vasc. Interv. Radiol., vol. 17, no. 12, pp. 1999-2004, Dec. 2006

3. B.M. Stoeckelhuber et al., "Radiation Dose to the Radiologist's Hand during Continuous CT FluoroscopyGuided Interventions," Cardiovasc. Intervent. Radiol., vol. 28, no. 5, pp. 589-594, June 2005

4. R.D. Nawfel et. al., "Patient and Personnel Exposure during CT Fluoroscopy-Guided Interventional Procedures," Radiol., vol. 216, no. 1, pp. 180-184, July 2016

5. B. Van Every, R.J. Petty, "Measurements of Computed Tomography Radiation Scatter," Australasian Phys. Eng. Sci. Med., vol. 15, no. 1, pp. 15-24, Apr. 2005

6. B.M. Stoeckelhuber et al., "Radiation Dose to the Radiologist's Hand during Continuous CT FluoroscopyGuided Interventions," Cardiovasc. Interv. Radiol., vol. 28, no. 5, pp. 589-594, Aug. 2005

7. M. Salvado, M. López, J.J. Morant and A. Calzado et. al., "Monte Carlo Calculation of Radiation Dose in CT Examinations Using Phantom and Patient Tomographic Models," Rad. Prot. Dosim., vol. 114, no.1-3, pp. 364368, May 2005 